\title{
COMPETÊNCIAS EMOCIONAIS COMO DISPOSITIVO PARA INTEGRALIZAÇÃO DO CUIDADO EM SAÚDE: CONTRIBUIÇÕES PARA 0 TRABALHO INTERPROFISSIONAL
}

\author{
Michelle Almeida Silva \\ Universidade Federal da Paraíba - UFPB \\ Érika Leite da Silva Cardoso \\ Universidade Federal da Paraíba - UFPB \\ Tatjana Teresa de Lima Miranda \\ Universidade Federal da Paraíba - UFPB \\ Juliana Sampaio \\ Universidade Federal da Paraíba - UFPB
}

\begin{abstract}
Resumo
A interprofissionalidade promove o aperfeiçoamento da assistência integral à saúde. Porém, o trabalho em equipe, por vezes, é permeado por conflitos, os quais podem ser gerenciados através das competências emocionais. Esse estudo objetiva relatar uma experiência de desenvolvimento de competências emocionais para fortalecimento do processo de trabalho interprofissional, com vistas à integralidade do cuidado. $\mathrm{O}$ Programa de Educação pelo Trabalho em Saúde desenvolveu essa experiência em 2015, num serviço de média complexidade. Inicialmente, foi utilizado um jogo, visando explorar fragilidades do serviço, o qual possibilitou identificar o sentimento de inibição de muitos profissionais diante de situações do cotidiano laboral. Posteriormente, objetivando contornar tais inibições, atividades lúdicas foram realizadas por meio de vídeos, jogos e rodas de conversas, que promoveram diálogos interprofissionais, com foco nas competências empatia e assertividade, que resultaram em uma postura mais ativa e questionadora dos profissionais frente às necessidades do processo de trabalho da equipe.
\end{abstract}

Palavras-chave: papel profissional; desempenho profissional; assistência integral à saúde; empatia; assertividade.

\section{EMOTIONAL COMPETENCES AS DEVICE FOR THE INTEGRALIZATION OF HEALTH CARE: CONTRIBUTIONS TO THE INTERPROFESSIONAL WORK}

\begin{abstract}
The interprofessionality promotes the improvement of the full assistance of the health care. However, sometimes teamwork is surrounded by conflicts that can be managed through emotional competencies. This study aims to report an experience of promoting emotional competencies for the strengthen of the interprofessional work's process, aiming to achieve the integrality of care. The Programa de Educação pelo Trabalho em Saúde developed this experience in 2015, in a medium complexity service. Initially, a game was used to explore the weaknesses of the service, which allowed to identify the feeling of inhibition of many professionals in a daily work situation. Subsequently, to overcome these inhibitions, playful activities were done through videos, games, and conversations, that promoted interprofessional dialogues, focused on the empathy and assertiveness competences, that resulted in a more active and questioning posture of the professionals face to the team work's process needs.
\end{abstract}

Keywords: professional role; work performance; comprehensive health care; empathy; assertiveness. 


\title{
COMPETENCIAS EMOCIONALES COMO DISPOSITIVO PARA INTEGRALIZACIÓN DEL CUIDADO EN SALUD: CONTRIBUCIONES AL TRABAJO INTERPROFESIONAL
}

\begin{abstract}
Resumen
La interprofesionalidad promueve la mejora de la asistencia integral de la salud. Sin embargo, el trabajo en equipo, a veces, está rodeado por conflictos, que pueden ser administrados a través de las competencias emocionales. Este estudio tiene como objetivo informar una experiencia de desarrollo de las competencias emocionales para la consolidación del proceso de trabajo interprofesional, con el fin de alcanzar la integralidad del cuidado. El Programa de Educación por el Trabajo en Salud desarrolló esa experiencia en 2015, en un servicio de mediana complejidad. En el principio, se utilizó un juego para explorar las debilidades en el servicio, el cual posibilitó identificar el sentimiento de inhibición de muchos profesionales frente a situaciones del cotidiano laboral. En seguida, para superar estas inhibiciones, se realizaron actividades lúdicas a través de videos, juegos y ruedas de conversación, que promovieron diálogos interprofesionales, centrados en las competencias empatía y asertividad, que resultaron en una postura más activa y cuestionadora de los profesionales frente a las necesidades del proceso de trabajo del equipo.
\end{abstract}

Palabras clave: rol profesional; rendimiento laboral; atención integral de salud; empatía; asertividad.

\section{INTRODUÇÃO}

Dentre os princípios do Sistema Único de Saúde (SUS), talvez o mais desafiador seja proporcionar atendimento integral à população, num contexto de hegemonia do modelo de atenção à saúde mecanicista e fragmentado. A integralidade da atenção busca transformar esse modelo, por meio do uso de tecnologias leves que permitam promover práticas de cuidado mais humanizadas, voltadas principalmente para as necessidades de cada indivíduo (Kalichman \& Ayres, 2016).

Para tornar o processo de trabalho em saúde de fato integral, é necessário que os trabalhadores se envolvam diretamente no ato de cuidar, por meio da escuta, do vínculo, da confiança, do respeito e do acolhimento (Cunha \& Zagonel, 2008). Esse investimento relacional permite que os profissionais de saúde compreendam as particularidades de cada indivíduo (Merhy \& Feuerwerker, 2009). Merhy e Franco (2003) apontam que o cuidado ampliado requer o protagonismo de todos atores. Ou seja, gestores, profissionais e usuários do sistema de saúde devem sentir-se corresponsáveis para que a produção da saúde seja de forma integral, ética, humanizada e resolutiva.

Nesse contexto, torna-se necessário que as equipes de saúde se relacionem de forma interprofissional, construindo metas, ações e resultados em coletividade, com vistas a uma atenção interdisciplinar, holística e de qualidade (Santos et al., 2010). O trabalho interprofissional possibilita a troca de conhecimentos, respeitando o espaço e opinião de cada trabalhador (Cardoso, Corralo, Krahl, \& Alves, 2015), desde que a equipe interaja e esteja disposta a construir suas ações em conjunto, buscando a ressignificação das suas práticas 
de cuidado, através de processos de reflexão, análise e repactuação em equipe (Santos et al., 2010).

Este processo não é simples e está permeado por conflitos e tensões (Guebur, Poletto, \& Vieira, 2007). Para Valente e Monteiro (2016), as emoções desempenham um papel fundamental na gestão desses conflitos. Por isso, a fim de tornar essa interação menos desgastante, algumas competências que envolvam as relações interpessoais devem ser desenvolvidas para auxiliar nas relações de trabalho compartilhado. Dentre essas competências estão as emocionais, aqui compreendidas como um conjunto de capacidades comportamentais aprendidas pelo indivíduo diante das demandas de uma situação de interação social, incluindo as capacidades de comunicação, resolução de problemas, cooperação, empatia e assertividade (Formozo, Oliveira, Costa, \& Gomes, 2012).

Vale salientar que a competência emocional é uma capacidade aprendida baseada na inteligência emocional. Assim, para aprender uma competência emocional, precisa-se de certo nível de inteligência emocional (Barreira, 2014; Gregório, 2008), pois esta determina o potencial para aprender aptidões práticas e, por sua vez, a competência emocional mostra até que ponto desenvolveu-se esse potencial (Gregório, 2008).

A inteligência emocional permite a identificação das emoções, a possibilidade de avalia-las e, sobretudo, a capacidade geri-las, expressando-as de forma apropriada e efetiva. Assim, tendo em vista que a inteligência emocional proporciona a possibilidade de reagir e ter acesso aos sentimentos, facilitando a compreensão de si e dos outros, entende-se que esta capacidade é essencial nos contextos profissionais, de modo especial daqueles que para desenvolverem seus trabalhos necessitam da relação com outras pessoas (Tavares, 2013).

Tal realidade é vivenciada pelos profissionais de saúde, os quais precisam não somente da relação com o usuário, mas também com toda equipe multiprofissional, para que o cuidado seja efetivo. Portanto, quando estes profissionais colocam em prática as competências emocionais, estas facilitam as relações humanas que ocorrem no ambiente de cuidado/trabalho. Isto porque exercita-se a capacidade de escuta, de compreensão e de respeito às necessidades do outro, a partir da conduta de reconhecimento das emoções que estão à sua volta e do melhor envolvimento entre os profissionais, e destes com os usuários (Formozo et al., 2012).

Profissionais com elevados níveis de inteligência emocional possuem maior capacidade de entender os seus sentimentos, os atos, gestos e emoções dos outros, criando uma relação mais favorável ao trabalho em equipe, sendo capaz de responder de forma adequada mesmo em situações de stress (Aveleira, 2013; Tavares 2013). Aveleira (2013) ainda acrescenta que a inteligência emocional de 
um trabalhador está positivamente associada à sua satisfação com o trabalho, bem como com seu desempenho.

Por isso, tendo em vista que o desenvolvimento de competências emocionais contribui favoravelmente com as relações interprofissionais, bem como com o trabalho realizado pelos mesmo, o presente estudo objetivou relatar uma experiência de desenvolvimento de competências emocionais para fortalecer o processo de trabalho interprofissional, com vistas à integralidade do cuidado em um serviço de saúde do município da João Pessoa.

\section{MÉTODO}

Trata-se de um estudo descritivo, do tipo relato de experiência, fruto da vivência de um dos grupos do Programa de Educação para o Trabalho em Saúde (Pet-Saúde) da Universidade Federal da Paraíba (UFPB), em atividade no ano de 2015.

O Pet-Saúde foi um programa do Ministério da Saúde em parceria com o Ministério da Educação de apoio e suporte para os cursos de graduação em saúde repensarem suas práticas de ensino e formarem profissionais com perfis críticos e adequados às necessidades e às políticas de saúde do país (Diário Oficial da União, 2008). O programa teve como proposta promover educação em saúde através de momentos de ensino-aprendizado entre estudantes, profissionais de saúde, gestores e usuários do SUS (Paulino et al., 2013).

Dentre os vários grupos do Pet-Saúde desenvolvidos na UFPB, em parceria com a Secretaria Municipal de Saúde de João Pessoa, um deles trabalhou com o eixo Educação Permanente (Pet-EP), constituindo-se numa estratégia que possibilitou o questionamento das práticas profissionais e facilitou discussões relativas ao processo de trabalho em equipe, apostando na construção de uma rede de cuidado integral, acolhedora e resolutiva, capaz de atender demandas com responsabilização, qualidade, vínculo e humanização (Viana et al., 2015).

O grupo Pet-EP contou com cinco grupos inseridos em diversos serviços de saúde nos três níveis de atenção. Dentre eles, um atuou no Centro de Atenção Integral à Saúde do Idoso (CAISI), localizado no município de João Pessoa, e contou com o apoio de uma estudante de odontologia, uma de enfermagem, uma de medicina, uma preceptora com formação em fisioterapia e uma tutora com formação em psicologia.

O CAISI é um serviço de média densidade tecnológica que atende usuários a partir de sessenta anos e conta com uma equipe de saúde multiprofissional composta por nutricionistas, dentistas, auxiliares de saúde bucal, médicos, enfermeiros, técnicos de enfermagem, psicólogos, assistentes sociais, fisioterapeutas, farmacêuticos e fonoaudiólogos. Ao todo, a equipe é composta por 57 funcionários, cujas principais características consistem em predominância 
sexo feminino $(82,5 \%)$, vínculo empregatício efetivo pela prefeitura $(77,2 \%)$ e carga horária de trabalho de $20 \mathrm{hs} / \mathrm{semana}(36,8 \%)$.

A incorporação do grupo Pet-EP ao serviço visou compartilhar saberes, identificar potencialidades e dificuldades vivenciadas no cotidiano do processo de trabalho da equipe. A partir dessa integração com o serviço e utilizando a Educação Permanente como um dispositivo para mudanças nas práticas de saúde, baseadas na reflexão crítica sobre o cotidiano do trabalho de profissionais na rede de serviços (Almeida, Bizerril, Saldanha, \& Almeida, 2016), o Pet-EP junto com os profissionais e gestores do serviço, formularam estratégias que ajudassem a solucionar problemas vivenciados no processo de trabalho da equipe.

As atividades desenvolvidas aconteceram uma vez por mês, durante o turno da manhã, no período de agosto a dezembro de 2015. A primeira atividade proposta objetivou identificar potencialidades e fragilidades no processo de trabalho da equipe multiprofissional do serviço. Para isso, a equipe Pet-EP junto com a preceptora do CAISI, desenvolveu um jogo, denominado jogo das emoções, que buscou estimular os profissionais a expressarem suas percepções em relação ao seu cotidiano laboral. Nesse momento, disponibilizaram-se a participar 33 profissionais que estavam presentes no dia do desenvolvimento dessa atividade.

O jogo proposto buscava através de "emoticons" (carinhas que representavam emoções), apontar quais os sentimentos/estados emocionais dos profissionais em relação ao ambiente de trabalho, aos colegas de trabalho da mesma área e de outras áreas de conhecimento e, por fim, aos usuários que utilizavam o CAISI.

Durante o jogo, os profissionais deveriam responder a questões que estavam dispostas em cartas sobre a mesa. Além das cartas contendo perguntas, existiam cartas contendo diferentes desenhos de "emoticons" que poderiam representar o estado/sentimento do profissional em relação ao contexto indagado, alguns exemplos de perguntas realizadas foram: "Qual o seu sentimento predominante em relação ao CAISI?", "Qual o seu sentimento predominante em relação aos seus colegas de trabalho? ", "Qual o seu sentimento predominante em relação aos usuários do CAISI? ". Dentre os estados emocionais representados pelos "emoticons", que poderiam ser dados como resposta, estavam: feliz, triste, inseguro, desinteressado, furioso ou inibido.

A partir da análise das respostas dadas pelos profissionais no jogo das emoções, o grupo PET-Ep com o setor de psicologia do serviço propôs a realização de outras quatro atividades: uma roda de conversa, uma dinâmica de grupo (cestas das competências), um jogo de tabuleiro (Jogo do CAISI), para tentar trabalhar algumas competências emocionais com os profissionais, que 
poderiam responder as dificuldades emocionais então encontradas e por fim, houve uma reunião de fechamento das intervenções propostas.

A roda de conversa foi realizada na presença de todos os profissionais e contou com a parceria do setor de psicologia da instituição. A ação proposta convidou a equipe a refletir sobre umas das competências emocionais mais necessárias para uma boa relação entre as pessoas: a empatia. Nesse espaço, foi abordada sua importância através de um vídeo intitulado "Gentileza gera gentileza, ou o vírus da gentileza" (Minutos psíquicos, 2015 agosto 27).

$\mathrm{Na}$ sequência, buscando identificar o conhecimento preexistente dos profissionais em relação às competências técnicas e emocionais, deu-se início a uma dinâmica de grupo, denominada de cestas das competências. Nela, disponibilizaram-se a participar da brincadeira 31 profissionais do serviço, os quais foram postos em roda e apresentados a uma cesta contendo várias tarjetas com nomes de algumas competências emocionais (comunicação, saber ouvir, amor, respeito, empatia, ética, cordialidade, segurança) e outra com algumas competências técnicas (restauração dentária, aferição de pressão arterial, prescrição de medicamentos, avaliação psicológica, entrega de medicamentos). Em seguida, recomendou-se que o grupo retirasse das cestas algumas daquelas palavras e colocasse no quadro o que cada palavra representava para eles, se eram competências técnicas ou competências emocionais.

Em momento posterior, foi realizado um jogo de tabuleiro, chamado jogo do CAISI. De maneira lúdica, os profissionais foram colocados diante de situações-problemas fictícias e se posicionavam diante da mesma. A finalidade do jogo foi problematizar o cotidiano do serviço, estimulando os profissionais a assumirem uma postura mais ativa frente às situações-problemas comuns no dia a dia profissional, vivenciadas entre usuários, colegas de trabalho e gestão.

Dinamicamente, os profissionais jogavam em grupos, divididos por setores do serviço. O jogo foi aplicado em dez setores (farmácia, serviço social, psicologia, enfermagem, odontologia, fisioterapia, recepção, marcação, serviços gerais, nutrição e fonoaudiologia) envolvendo, ao todo, 22 profissionais que se dispuseram espontaneamente a participar da brincadeira.

Por fim, para realizar o fechamento destas atividades propostas, uma reunião foi marcada com os profissionais, a fim de proporcionar uma devolutiva dos resultados obtidos em todos os setores da instituição, bem como promover um diálogo na presença de todos os trabalhadores. Para tanto, a sala de reunião foi organizada de modo que todas as situações-problemas contidas no jogo do CAISI estavam dispostas em cartazes espalhados pelas paredes e as respostas obtidas dos diversos setores do CAISI estavam em forma de tarjetas, misturadas e coladas em um quadro branco.

A reunião iniciou com a apresentação de um vídeo intitulado "Agressivo, passivo ou assertivo?" (Minutos psíquicos, 2015 dezembro 14), visando diferenciar diferentes posturas que podem ser reproduzidas no ambiente de 
trabalho, bem como mostrar a importância do posicionamento assertivo da equipe em prol da construção coletiva de consensos para enfrentamento dos problemas. Em seguida, os profissionais foram convidados a colocar abaixo dos cartazes com as situações-problemas, as respectivas respostas de enfrentamento dos problemas que foram obtidas durante o jogo do CAISI e que estavam fixadas no quadro branco. Após esse momento, a partir de uma roda de conversa, passou-se a problematizar as diversas respostas dadas para cada situaçãoproblema vivenciada no dia a dia do CAISI.

Todas as atividades realizadas foram processadas, discutidas e analisadas em reuniões semanais da equipe do Pet-Ep e sua tutora. Estas analises foram fundamentadas nos referenciais teóricos da educação permanente em saúde e das competências emocionais.

Este trabalho respeita as diretrizes éticas da resolução 510/2016 e, por se tratar de um relato de experiência realizado interinstitucionalmente entre os membros do programa Pet-Saúde, eixo Educação Permanente e os profissionais do CAISI, não foi necessário a aprovação pelo comitê de ética em pesquisa. No entanto, foi respeitada a confidencialidade dos resultados obtidos e a autonomia dos profissionais na participação das atividades desenvolvidas.

\section{RESULTADOS E DISCUSSÃO}

Os momentos iniciais de integração das estudantes do Pet-EP com os profissionais da instituição foram de extrema relevância. Eles permitiram conhecer a dinâmica e o processo de trabalho da equipe, assim como possibilitaram a formação de vínculo com os profissionais da instituição. Por meio dessa experiência, as dificuldades da equipe foram sendo identificadas, fundamentando a proposição de atividades vinculadas a proposta do Pet-EP, cujo objetivo consistia em fazer com que os profissionais pudessem problematizar seus processos de trabalho.

No momento inicial, os resultados obtidos a partir da primeira atividade, denominada jogo das emoções, indicaram que $45.5 \%$ dos profissionais consideravam-se felizes em relação ao ambiente de trabalho. No entanto, 33.3\% dos profissionais apontavam um estado de inibição quanto a mesma proposição. No que tange aos sentimentos dos profissionais em relação ao seu setor e colegas da mesma profissão, 31\% afirmaram-se inibidos e 31\% afirmaram sentir-se feliz. Quanto a relação interprofissional, mais da metade deles, precisamente $51.7 \%$, também apontaram o sentimento de inibição. Por fim, em relação aos usuários, $66.7 \%$ afirmaram sentir-se felizes.

Desse modo, a partir dessa realidade, foi possível perceber que a maioria dos profissionais se sentiam felizes em relação aos itens apresentados. No entanto, um número relevante trouxe à tona o sentimento de inibição em relação ao ambiente de trabalho, aos colegas de profissão e ao relacionamento 
interprofissional. Ao mesmo tempo, identificou-se que o estado de inibição apontado por parte dos entrevistados parecia gerar uma imparcialidade que os impedia de desenvolver sua capacidade de assertividade, prejudicando, assim, seus processos de trabalho. Para Tavares (2013), indivíduos que apresentam boa gestão emocional expressam o que pensam e agem em conformidade com os seus sentimentos, ao invés de agirem de acordo com o que os outros querem ou esperam deles.

Nesse sentido, ficou clara a dificuldade de relacionamento entre a equipe, sobretudo, com aqueles que não estavam na mesma área de atuação. Tal dificuldade gerava como consequência a fragmentação do cuidado, comprometendo a integralidade do mesmo. Assim, era necessário que a equipe produzisse formas de interagir e de se relacionar mais participativas e democráticas, levando em consideração a importância de todos no processo do cuidado. Cada um com sua especificidade, respeitando o conhecimento do outro (Silva et al., 2013).

Se existe dificuldade no trabalho interprofissional, há necessidade de qualificar o envolvimento relacional da equipe. Para tanto, os profissionais devem aperfeiçoar suas habilidades para lidar com as emoções que os cercam. Portanto, desenvolver a inteligência emocional é imprescindível para tornar as relações sociais plenas e satisfatórias, uma vez que essa aptidão favorece relações interprofissionais mais cooperativas e interativas (Goleman, 2011).

Desse modo, para desenvolver as competências emocionais e colocá-las em prática, o profissional necessita compreender suas próprias emoções e as que estão a sua volta, favorecendo o diálogo e as interações interpessoais, tornando a equipe mais comunicativa, equilibrada, articulada e produtiva, bem como o ambiente de trabalho mais estimulante, saudável e menos conflitante (Guebur, et al., 2007).

Fortalecer essas habilidades de autoanálise das emoções pode transformar o trabalho em equipe, uma vez que o uso das competências emocionais permite que o indivíduo tenha capacidade de escutar o que o outro tem a dizer, bem como se posicionar em situações que requerem exposição de novas ideias e opiniões (Weisinger, 2001). Por isso, as competências emocionais são essenciais para a realização do trabalho de forma mais satisfatória, plena e harmoniosa, pois a comunicação facilita as tomadas de decisões para resoluções de problemas (Paschoal, Torres, \& Barreiros, 2010).

Nesse sentido, as atividades subsequentes propostas pelo Pet-Ep foram estruturadas de forma que buscassem estimular o desenvolvimento de algumas competências emocionais. Sabe-se que tais competências não são muito trabalhadas no currículo de formação em saúde, mas são extremamente necessárias para um bom desenvolvimento das relações interpessoais (Guebur, et al., 2007). A partir dessa concepção, a sequência das dinâmicas de trabalho do Pet-Ep buscou proporcionar uma experiência fundamentalmente 
horizontalizada, promovendo o protagonismo dos seus participantes, fazendo-os problematizar o cotidiano do seu processo de trabalho, bem como, utilizar suas competências emocionais para enfrentamento das realidades inerentes ao contexto laboral. Portanto, as outras atividades desenvolvidas tiveram o intuito de motivar os profissionais quanto à duas competências emocionais: empatia e assertividade.

Nesse contexto, na segunda ação do grupo, a ideia foi realizar um momento de reflexão sobre empatia. Através do vídeo "Gentileza gera gentileza" foi possível sensibilizá-los quanto à necessidade de colocar-se no lugar do outro, buscando compreender suas aflições e tentando, através de pequenas atitudes, ajudá-lo.

A empatia nada mais é que a capacidade de tomar consciência dos sentimentos, necessidades e preocupações dos outros, colocando-se no lugar do mesmo, estabelecendo laços e promovendo sentimentos de partilha (Tavares, 2013). Segundo Domingues (2009), no contexto de trabalho, a empatia envolve dar importância aos sentimentos do outro e considerá-los para tomadas de decisões mais eficazes.

Pequenos gestos de empatia podem também ser chamados de atos de gentileza, esses gestos podem materializar-se no cotidiano através de uma atitude, palavra ou pensamento cordial que conduz a um sentimento de atenção e preocupação com outro. Ser gentil promove um sentimento de bem-estar mútuo e, muitas vezes, gera um ciclo que desencadeia uma corrente de bondade (Koval, \& Kaplan, 2008).

Portanto, a empatia pode transformar as relações entre pessoas, pois transmite respeito, educação, acolhimento, preocupação, cuidado. Essas características são fundamentais em qualquer tipo de relação humana e, por isso, devem estar presentes, sobretudo, na área da saúde, em que os profissionais interagem diretamente com seres humanos que estão fragilizados ou debilitados, necessitando de atenção.

A intenção da terceira dinâmica desenvolvida foi construir junto com a equipe o conceito dessas competências e diferenciá-las, através da dinâmica: cestas das competências. Além disso, buscou-se refletir sobre o processo de trabalho e a importância do uso das competências emocionais. Ao término da atividade, observou-se uma participação muito tímida da equipe quanto ao protagonismo na atividade, com forte predomínio da fala da gestora do serviço. Notou-se ainda que a presença da gestora na atividade gerou inibição por parte dos profissionais durante a dinâmica, prejudicando, de certo modo, a atividade.

O diálogo verticalizado pela hierarquia prejudica o andamento dos processos de trabalho. A equipe deve ser capaz de se posicionar democraticamente, de forma que todos possam participar, construindo diálogos, operacionalizando ideias, articulando conhecimentos, com objetivos em comum (Amestoy et al., 2013; Morreti-Pires, \& Campos, 2009). Por isso, para 
Domingues (2009) pessoas com alto nível de inteligência emocional são as mais adequadas para assumirem cargos de liderança, pois constroem relações interpessoais mais sólidas e proporcionam um funcionamento mais saudável da equipe.

Nesse sentido, para vencer essa barreira e permitir que os profissionais se posicionassem livremente e de forma horizantalizada, a equipe foi conduzida a refletir sobre uma segunda competência emocional: a assertividade. Tal competência consiste em comunicar-se de maneira franca, permitindo ao profissional a possibilidade de posicionar-se abertamente e opinar em situações do cotidiano do trabalho. A falta de assertividade é capaz de gerar muitos conflitos e mal-entendidos, pois muitas vezes as opiniões pessoais são reprimidas em uma discussão, simplesmente por falta de coragem de posicionarse como gostaria (Marchezini-Cunha, \& Tourinho, 2010).

Levando em consideração a falta de assertividade durante os momentos de dinâmica em equipe, percebeu-se a necessidade de trabalhar essa competência emocional de maneira particular. Nesse sentido, a quarta atividade deu-se através do jogo do CAISI. O jogo foi um momento significativo nesse processo de reflexão sobre assertividade, pois tornou-se um dispositivo que permitiu o diálogo espontâneo entre os profissionais que, à medida que brincavam, se posicionavam e utilizavam daquele espaço para colocar o que sentiam, suas angústias, justificativas, além de problematizarem o processo de trabalho dos colegas e o seu. Ao término da atividade, percebeu-se que a mesma alcançara seu objetivo: estimular a assertividade, a fim de fazer com que os trabalhadores do CAISI se posicionassem de forma ativa frente aos problemas do serviço.

Na reunião de fechamento e avaliação das atividades desenvolvidas, foi possível notar que a maioria dos profissionais, com algumas exceções, relataram as situações que os incomodavam, realidade não vivenciada no início de nossa ação do Pet-EP. A presença da gestão, antes centralizadora, não mais os intimidava e, com respeito e diálogo, todos expuseram suas opiniões e seus pontos de vista, exercitando, assim, uma postura assertiva, ativa e questionadora.

Alguns dos profissionais relataram que a maioria dos que ali estavam se encontravam acomodados e, por isso, se comportavam de maneira passiva, realizando apenas sua função burocraticamente definida. Por isso, desenvolviam pouca pró-atividade diante das situações. Contudo, afirmaram que as ações propostas pelo Pet-EP serviram de estímulo para que pudessem despertar para o agir. O estado de passividade foi enfraquecido e o pensamento crítico e reflexivo passou a estar mais presente durante o enfrentamento dos problemas, a fim de obter as mudanças desejadas pelo coletivo. 


\section{CONSIDERAÇÕES FINAIS}

A experiência do Pet-EP no CAISI promoveu o desenvolvimento de competências emocionais fundamentais para o alívio de tensões e conflitos que permeavam as relações interprofissionais na saúde. Essas competências, apesar de pouco trabalhadas nos diversos níveis de formação profissional, marcadas pela valorização da técnica, são imprescindíveis para promover o cuidado em saúde humanizado, integral, resolutivo e de qualidade.

As atividades propostas permitiram a problematização do processo de trabalho de forma democrática e horizontalizada, protagonizadas por todos os profissionais. Essas ações proporcionaram aos trabalhadores a percepção da importância da interação interprofissional para a integralização do cuidado.

Os profissionais também notaram que o trabalho quando é realizado em coletividade necessita do uso das competências emocionais, como empatia e assertividade, para aprimorar as relações interpessoais e promover o bem-estar psicológico da equipe, ressignificando as práticas de cuidado, tornando-as mais acolhedoras, humanas, reflexivas e questionadoras.

Nesse contexto, a abertura de espaços dialógicos, através da educação permanente, foi indispensável para permitir que os trabalhadores se expressassem livremente e alcançassem uma postura mais ativa e questionadora perante os problemas do cotidiano do serviço e dos pensamentos enrijecidos da equipe.

Como limitações da experiência, é possível ressaltar a dificuldade de envolver todos os profissionais do serviço, visto que nem todos estavam presentes nos dias de desenvolvimento das atividades pelo grupo Pet-Ep. Além disso, não houve uma adesão dos médicos da equipe na participação das reuniões e atividades coletivas. Outra limitação está no fato de que a equipe deve dar continuidade aos processos avaliativos das suas relações afetivas e interpessoais de maneira autônoma, após o término da vigência de atuação do programa Pet-Ep no CAISI. Desta forma, os profissionais devem instituir reuniões sistemáticas de autoanálise do cotidiano laboral, para que seja possível que outras competências emocionais sejam trabalhadas e os resultados obtidos dessa experiência perdurem e sejam aprimorados.

\section{DECLARAÇÃO DE CONFLITO DE INTERESSES}

Não há conflito de interesses. 


\section{REFERÊNCIAS}

Almeida, J. R. S., Bizerril, D. O., Saldanha, K. G. H., \& Almeida, M. E. L. (2016). Educação permanente em saúde: Uma estratégia para refletir sobre o processo de trabalho. Revista ABENO, 16(2), 7-15.

Amestoy, S. C., Backes, V. M. S., Thofehrn, M. B., Martini, J. G., Meirelles, B. H. S., \& Trindade, L. L. (2013). Percepção dos enfermeiros sobre seu processo de ensino aprendizagem da liderança. Texto \& Contexto Enfermagem, 22(2), 468-475. doi:10.1590/S0104-07072013000200024

Aveleira, J. J. C. B. (2013). A inteligência emocional, o desempenho e a satisfação laboral em funções comerciais. (Dissertação de Mestrado). Instituto Universitário de Ciências Psicológicas, Sociais e da Vida, Lisboa.

Barreira, L. N. (2014). Inteligência emocional: Associação com job engagement em enfermeiros no contexto da oncologia. (Dissertação de Mestrado). Universidade Lusófona de Humanidades e Tecnologias, Lisboa.

Cardoso, A. C., Corralo, D. J., Krahl, M., \& Alves, L. P. (2015). O estimulo à prática da interdisciplinaridade e do multiprofissionalismo: A extensão universitária como uma estratégia para a educação interprofissional. Revista ABENO, 15(2), 12-19.

Cunha, P. J., \& Zagonel, I. P. S. (2008). As relações interpessoais nas ações de cuidar em ambiente tecnológico hospitalar. Escola Paulista de Enfermagem, 21(3), 412-419. doi:10.1590/S0103-21002008000300005

Diário Oficial da União. (2008, agosto 27). Portaria Interministerial no 1.802 Programa de Educação pelo Trabalhador para a Saúde - PET Saúde. Recuperado de: http://bvsms.saude.gov.br/bvs/saudelegis/gm/2008/pri1802_26_08_2008. html

Domingues, A. R. S. (2009). Inteligência emocional, empatia e satisfação no trabalho em médicos. (Dissertação de Mestrado). Universidade do Porto, Porto.

Formozo, G. A., Oliveira, D. C., Costa, T. L., \& Gomes, A. M. T. (2012). As relações interpessoais no cuidado em saúde: Uma aproximação ao problema. Revista Enfermagem UERJ, 20(1), 124-127. Recuperado de: http://www.epublicacoes.uerj.br/index.php/enfermagemuerj/article/view/40 $06 / 2775$.

Goleman, D. (2011). Inteligência emocional: A teoria revolucionária que define o que é ser inteligente. Rio de Janeiro, RJ: Objetiva.

Guebur, A. Z., Poletto, C. A., \& Vieira, D. M. S. (2007). Inteligência emocional no trabalho. Revista Intersaberes, 2(3), 71-96.

Gregório, F. A. S. (2008). Competência emocional e satisfação profissional dos enfermeiros. (Dissertação de Mestrado). Universidade do Algarve, Algarve. 
Kalichman, A. O., \& Ayres, J. R. C. M. (2016). Integralidade e tecnologias de atenção: Uma narrativa sobre contribuições conceituais à construção do princípio da integralidade no SUS. Caderno de Saúde Pública, 32(8), 1-13. doi: 10.1590/0102-311X00183415

Koval, R., \& Kaplan, L. T. (2008). O poder da gentileza: Descubra como a simpatia e a delicadeza podem transformar sua vida. Rio de Janeiro, RJ: Sextante.

Marchezini-Cunha, V., \& Tourinho, E. Z. (2010). Assertividade e autocontrole: Interpretação analítico-comportamental. Psicologia Teoria e Pesquisa, 26(2), 295-304. doi:10.1590/S0102-37722010000200011

Merhy, E. E., \& Feuerwerker, L. C. M. (2009). Novo olhar sobre as tecnologias de saúde: Uma necessidade contemporânea. In A. C. S. Mandarino, \& E. Gomberg, Leituras e Novas Tecnologias e Saúde (pp. 29-74). São Cristóvão, SE: Editora UFS.

Merhy, E. E., \& Franco, T. B. (2003). Por uma composição técnica do trabalho centrada nas tecnologias leves e no campo relacional e nas tecnologias leves. Apontando mudanças para os modelos tecnoassistenciais. Revista Saúde em Debate, 27(65), 316-327.

Minutos psíquicos. (2015, agosto 27). Gentileza gera gentileza, ou o vírus da gentileza [Video file].

Recuperado de: https://www.youtube.com/watch?v=nexOPbgQkHk

Minutos psíquicos. (2015, dezembro 14). Agressivo, passivo ou assertivo? [Video file]. Recuperado de: https://www.youtube.com/watch?v=rd1mCZVNnxE

Morreti-Pires, R. O., \& Campos, D. A. (2010). Equipe multiprofissional em saúde da família: do documental ao empírico no interior da Amazônia. Revista Brasileira de Educação Médica, 34(3), 379-389. doi:10.1590/S010055022010000300007

Paschoal, T., Torres, C. V., \& Barreiros, J. P. (2010). Felicidade no trabalho: Relações com suporte organizacional e suporte Social. Revista de Administração Contemporânea, 14(6), 1054-1072. doi:10.1590/S141565552010000700005

Paulino, H. H., Souza, P., Codato, L. A. B., Muraguchi, E. M. O., Higasi, M. S., Casaroto, P., \& Raminelli, M. (2013). Grupo de gestantes: Uma estratégia de intervenção do PET-Saúde da Família. Revista ABENO, 13(2), 76-81.

Santos, R. R., Lima, E. F. A., Freitas, P. S. S., Galavote, H. S., Rocha, E. M. S., \& Lima, R. C. D. (2016). A influência do trabalho em equipe na atenção primária à saúde. Revista Brasileira de Pesquisa em Saúde, 18(1), 130-139.

Silva, P. A., Silva, G. M. L., Rodrigues, J. D., Moura, P. V., Caminha, I. O., \& Ferreira, D. K. S. (2013). Atuação em equipes multiprofissionais de saúde: Uma revisão sistemática. Conscientiae Saúde, 12(1), 153-160. doi: $10.5585 /$ conssaude.v12n1.3987 
Tavares, L. (2013). Inteligência emocional e a satisfação no trabalho. (Dissertação de Mestrado). Universidade Lusófona de Humanidades e Tecnologias, Lisboa.

Valente, M. N., \& Monteiro, A. P. (2016). Inteligência emocional em contexto escolar. Revista Eletrônica de Educação e Psicologia, 7, 1-11.

Viana, D. M., Araujo, R. S., Vieira, R. M., Nogueira, C. A., Oliveira, V. C., \& Renno, H. M. S. (2015). A educação permanente em saúde na perspectiva do enfermeiro na estratégia de saúde da família. Revista de Enfermagem do Centro Oeste Mineiro, 5(2), 1658-1668. doi:10.19175/recom.v0i0.470

Weisinger, H. (2001). Inteligência emocional no trabalho: Como aplicar os conceitos revolucionários da I. E. nas suas relações profissionais, reduzindo o estresse, aumentando sua satisfação, eficiência e competitividade. Rio de Janeiro, RJ: Objetiva.

\section{Sobre as autoras}

Michelle Almeida Silva é mestranda em Ciências Odontológicas pela Universidade Federal da Paraíba (UFPB) e-mail: michellealmeidasilva@hotmail.com

Érika Leite da Silva Cardoso é enfermeira pela Universidade Federal da Paraíba (UFPB). E-mail: erika-Isc@hotmail.com

Tatjana Teresa de Lima Miranda é fisioterapeuta pela Universidade Federal da Paraíba (UFPB), trabalha no Centro de Atenção Integral à Saúde do Idoso (CAISI). E-mail: tlimamiranda@gmail.com Juliana Sampaio é psicóloga pela Universidade Federal de Pernambuco (UFPE), Mestre em Serviço Social pela Universidade Federal de Pernambuco (UFPE), Doutora em Saúde Pública pela Fundação Oswaldo Cruz. E-mail: julianasmp@hotmail.com

A contribuição de cada autora pode ser atribuída como se segue: Michelle Almeida Silva, Érika Leite da Silva Cardoso, Tatjana Teresa de Lima Miranda e Juliana Sampaio contribuíram para a conceitualização, investigação e visualização do artigo; Michelle Almeida Silva, Érika Leite da Silva Cardoso, Tatjana Teresa de Lima Miranda e Juliana Sampaio fizeram a redação inicial do artigo (rascunho) e Michelle Almeida Silva, Érika Leite da Silva Cardoso, Tatjana Teresa de Lima Miranda e Juliana Sampaio são os responsáveis pela redação final (revisão e edição).

Os autores agradecem ao Ministério da Saúde pelo financiamento do programa PET-Saúde que resultou na elaboração deste trabalho.

Recebido em: 03/12/2017

$1^{\text {a }}$ revisão em: $16 / 03 / 2018$ Aceito em: $15 / 04 / 2018$ 